\title{
Analysis And Improvement Of Leaked Failure For Automotive Water Pump
}

\author{
Zhanlong Cao ${ }^{1}$, Xingxing X ${ }^{2}$, Qunpo Zhang ${ }^{2}$, Fengmin Zhang ${ }^{1}$ \\ 1.School of Mechanical Engineering, Hubei University of Automotive Technology, Shiyan, \\ 442002, China 2. Dongfeng Motor Pump Company Limited, Shiyan, 442001, China
}

Keywords: automotive water pump; thermostat cover; failure analysis; improvement

\begin{abstract}
A model automotive pump was studied, and the leaked failure of automotive water pump was analyzed. Through the statistical data, it was found the failure is mainly due to leakages of thermostat cover. Therefore, the reason of leakages of thermostat cover was analyzed, and improvement scheme was proposed. The scheme was discussed and validated. Finally, the scheme to increase seal gasket of thermostat cover is considered to be the best solution. Product testing and mass production shows that the leakages of thermostat cover don't appear. The leaked failure of automotive water pump is reduced.
\end{abstract}

\section{Introduction}

Automotive water pump is the core component of automobile engine cooling system. Its role is to improve the working pressure of the cooling fluid and maintain coolant circulation between engine-related components, which takes away the heat generated by the engine work ${ }^{[1]}$. If the automotive water pump leaks, it will directly affects the engine performance, which seriously affects the cooling system's stability and reliability. Therefore, a type of car water pump leakage situation is analyzed. Leakages of automotive water pump causes are analyzed and improved. Leakage of the fault is reduced and performance of the car engine has been improved.

\section{Analysis of leaked failure of automotive water pump}

\subsection{Introduction to automotive water structure}

The automotive water pump to be studied is a centrifugal pump, which widely used in automotive engines ${ }^{[2,3]}$. The engine drives the pump shaft and the pump impeller through the transmission gear. The coolant in the pump is driven by the impeller. In the role of centrifugal force is thrown to the edge of the pump shell, while generating a certain pressure. This pressure causes the coolant to flow out of the waterway or water pipe. The pump and thermostats are integrated. The pump is mainly composed of pump housing, pump shaft, seals, pump impeller, transmission gear, pump cover, thermostat, and thermostat cover. The specific structure is shown in Figure 1.

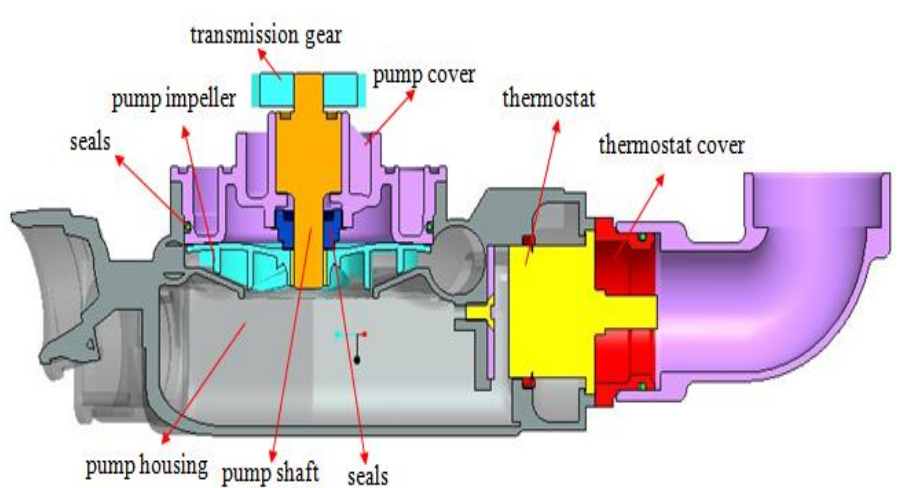

Figure 1: Schematic diagram of automotive water pump

\subsection{Analysis of leaked failure of automotive water pump type}

Pump leakage fault element is collected while collecting member is disassembled. The total number of pump leaks is 118 pieces. The cause of the leak was analyzed statistically, as shown in figure 2.As can be seen from the statistical results, The main reason for the automotive water pump leak is. The main reason for the automotive water pump leak is leakage of thermostat cover, pump cover seals leaks, water seal leak and other reasons. The leakage of thermostat cover accounts for $49.3 \%$. Its proportion is relatively high. Therefore, it is necessary to further analyze the causes of leakage of thermostat cover ${ }^{[4]}$.

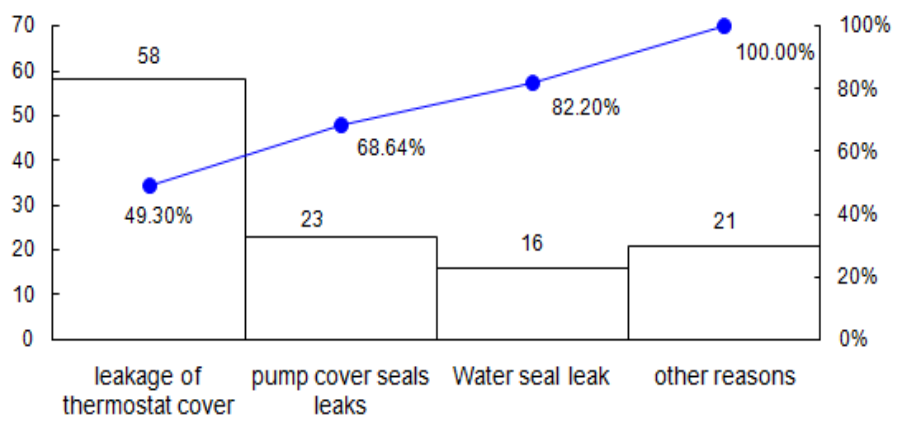

Figure 2: Distribution of Leakage faults in automotive water pump 


\subsection{Analysis of the leakages of thermostat cover}

The causes of leakages of thermostat cover are analyzed from the view of processing size, sealing material and design structure. The depth of recess of pump housing and the roughness are measured, as shown in figure 3 . The results show the depth range of 5.42-5.48 $\mathrm{mm}$. The size of the qualified range is $5.35-5.65 \mathrm{~mm}$, which is in line with the requirements. The roughness measurement is $\mathrm{Ra}=2.43 \mu \mathrm{m}$, which is in line with the requirements.

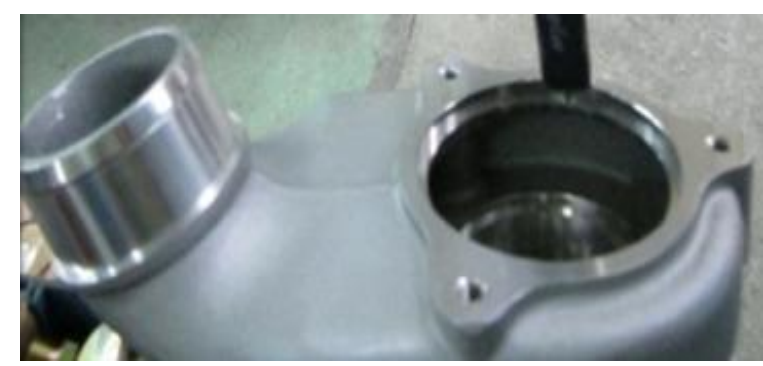

Figure 3: Recess of pump housing

The size of the thermostat seal is measured, as shown in figure 4 . The results show the thickness range of 6.65-6.76 $\mathrm{mm}$. The size of the qualified range is $6.6-6.9 \mathrm{~mm}$, which is in line with the requirements.

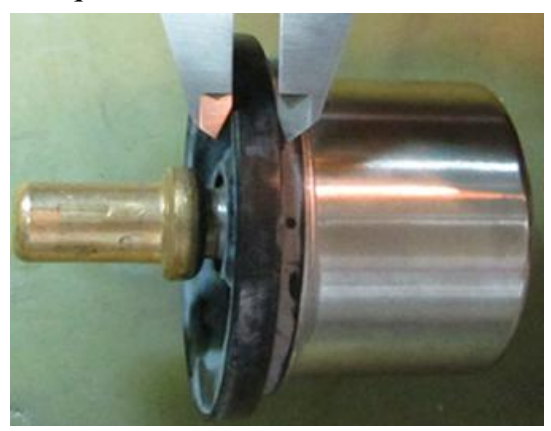

Figure 4: thermostat seal

From the design point of view, the end of the thermostat seal with rubber seal ${ }^{[5]}$, this is dependent on the compression of rubber to seal. This means that the seal is entirely dependent on the rubber anti-aging ability, As shown in Figure 5. Once the rubber is aged, it will make the sealing effect worse or even fail. The disassembled components are observed, as shown in Figure 5. The sealing bosses have been polished and the rubber has lost the elasticity of the seal. Therefore, this is the main reason for the formation of leakage, which requires the improvement of this part of the design.

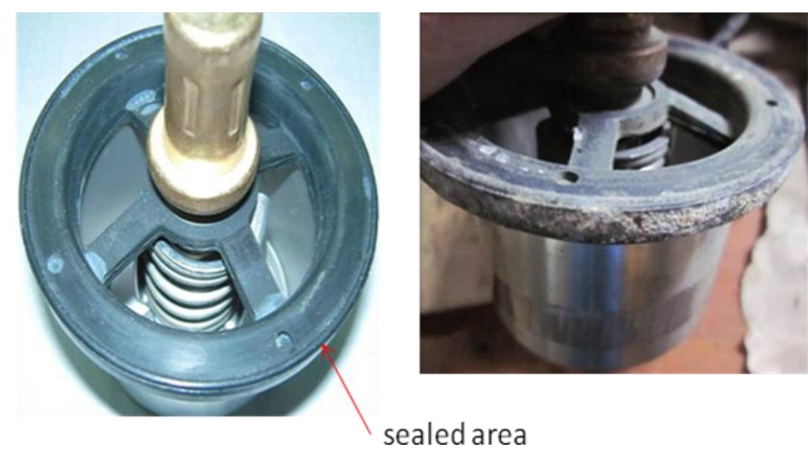

Figure 5: Sealed area and fault pieces

\section{Improvement of automotive water pump}

\subsection{Scheme analysis}

For the aging of the rubber problem, we can be resolved from three aspects. First, we can reduce the aging by replacing the better thermostat rubber material. Now the material EPDM rubber is used. The material properties are characterized by moderate price, heat, and rebound rate slightly worse, easy to aging. The price of EPDM rubber is 4000 Yuan / ton. Through the use of better materials NBR nitrile rubber, Use it to replace EPDM rubber. Its material performance is characteristics of expensive, heat-resistant, good resilience, anti-aging ability. However, the price is really expensive. The price of EPDM rubber is 13500 Yuan / ton. If this will greatly increase the cost of the product is not conducive to market competition, the program is not desirable.

Second, in the thermostat cover contact surface to increase the pneumatic coating, as shown in figure 6.The use of pneumatic coating machine, in the thermostat cover contact surface coating, which can reduce the leakage, but it is easy to create new problems. Such as operating trouble, the amount of glue is not easy to control; the appearance is not good, affecting painting. The program is still not feasible.

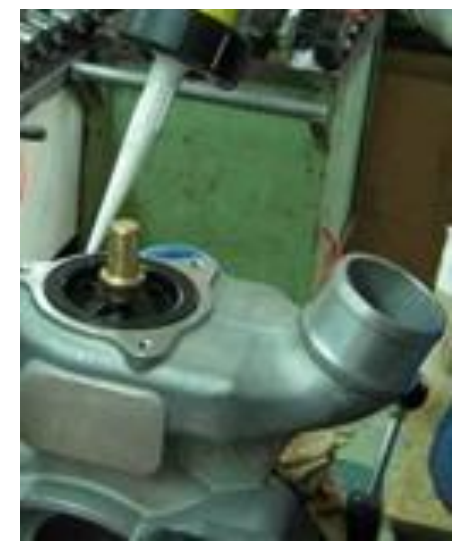

Figure 6: Increase the pneumatic coating

Third, increasing seal gasket design to the thermostat cover. This program is low cost, and easy to operate control, the program is feasible.

\subsection{Increase seal gasket design}

First, we can consider increasing the pad to increase the paper pad, which increases the so-called gasket in the gasket. That is what we usually call rubber asbestos board. After confirming that the paper pad needs to be intrinsically damaged before the installation, the operation is not easy to control. And the pad can withstand the pressure below the thermostat cover parts must bear the pressure value, prone to collapse. So the use of such paper pads is not appropriate, the program is not feasible.

Second, we can consider adding foam rubber metal composite plate gasket. The characteristic of foam rubber metal composite panel gasket is the surface rubber NBR. This 
material in the pump working load (34.5MPa).Compression rate of $35 \pm 10 \%$, recovery rate $\geqslant 65 \%$,Application: Coolant temperature $-40{ }^{\circ} \mathrm{C}-+150{ }^{\circ} \mathrm{C}$. And pump operating conditions under the material requirements are consistent. The program is feasible. The structure is shown in Figure 7.

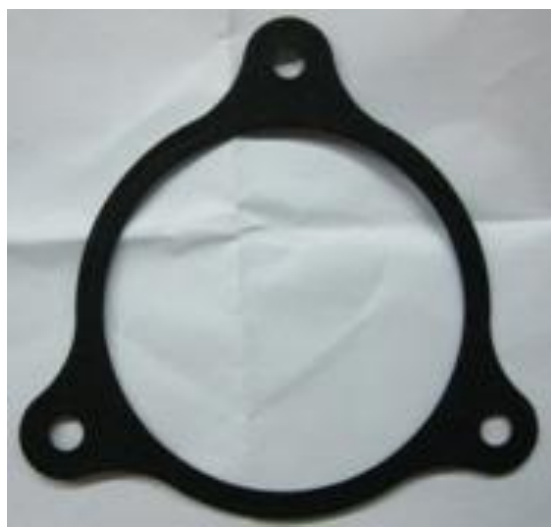

Figure 7: Foam rubber metal composite plate gasket structures

Seal diameter size selection thermostat diameter: 74 (-0.3 to + $0.1) \mathrm{mm}$, the outer diameter of the gasket is selected as the size of the volute: $74.5(0 \sim+0.2) \mathrm{mm}$, thickness of $0.8 \pm$ $0.08 \mathrm{~mm}$. In order to prevent axial movement of the thermostat, the groove depth of the volute in contact with the thermostat rubber is changed from $5.5 \pm 0.15 \mathrm{~mm}$ to 5.5 (+ $0.15 / 0) \mathrm{mm}$. The gasket is installed in the pump, as shown in figure 8 .

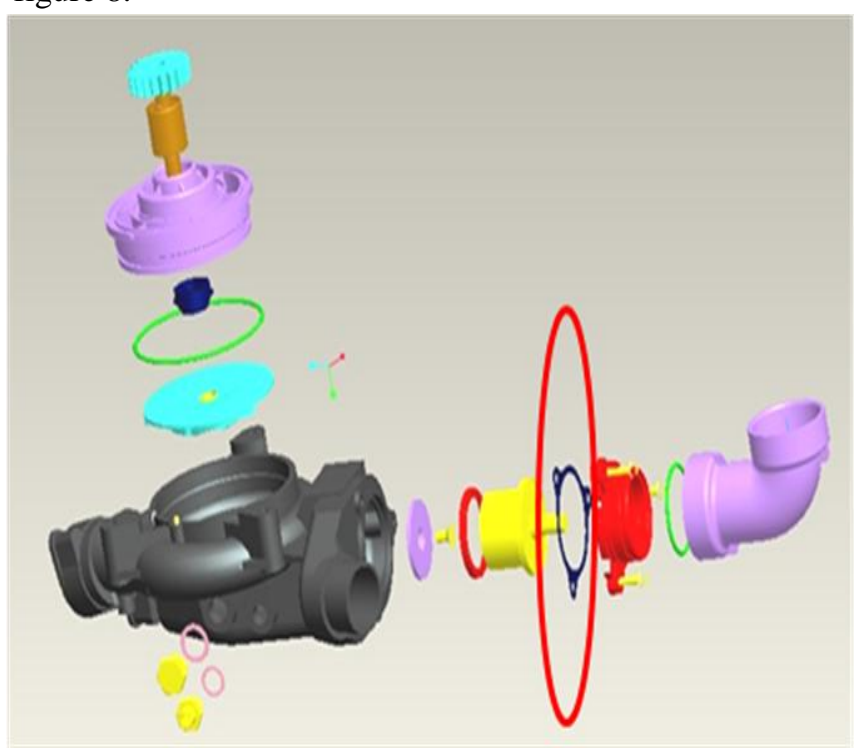

Figure 8: The installation position of the gasket in the pump

\subsection{Seal pressure check}

The bolt mounting position is shown in Figure 9.Bolts use M6 bolts, the material is 35 , the yield strength is $640 \mathrm{Mpa}$, and the torque is $10 \mathrm{~N} . \mathrm{m}$.

Calculated,

$$
\mathrm{F}_{0} \leqslant(0.6-0.7) * 640 * 3 * 3=10857 \sim 12667 \mathrm{~N}
$$

The relationship between preload and preload torque:

$$
\mathrm{T}=0.2 * \mathrm{~F}_{0} * \mathrm{~d}
$$

d---bolt nominal diameter

T---wrench tightening torque

$\mathrm{F}_{0}=\mathrm{T} / 0.2 * \mathrm{~d}=10 * 1000 / 0.2 * 6=8333.3 \mathrm{~N}$

The single face surface area of the gasket is $2172 \mathrm{~mm} 2$, and the gasket is subjected to the pressure.

$3 * 8333.3 \mathrm{~N} / 2172 \mathrm{~mm} 2=11.51 \mathrm{MPa}$

The pressure of the gasket provided by the manufacturer is up to $34.5 \mathrm{Mpa}$. The current design meets the requirements.

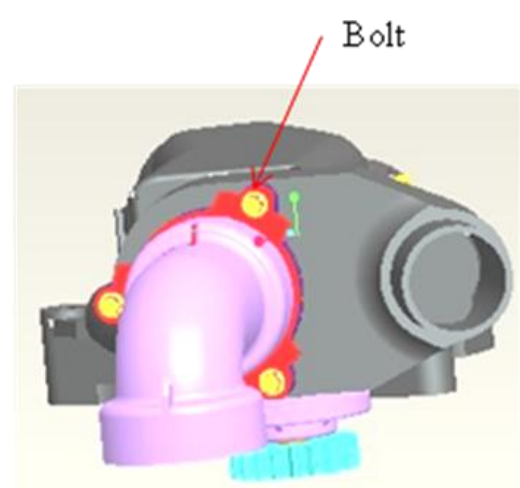

Figure 9: Bolt mounting position

\section{Improvement effect}

The improved pump was carried out a number of levels of sealing test ${ }^{[6,7,8]}$, the test results shown in Table 1 . From the test results, the end of the thermostat cover leakage situation did not leak.

\begin{tabular}{|l|c|l|l|}
\hline \multicolumn{1}{|c|}{ type } & $\begin{array}{c}\text { speed } \\
\text { reliability test }\end{array}$ & $\begin{array}{l}\text { durability } \\
\text { test of } \\
\text { commercial } \\
\text { vehicle } \\
\text { technology } \\
\text { center }\end{array}$ & $\begin{array}{l}\text { reliability } \\
\text { Test of } \\
\text { commercial } \\
\text { vehicle } \\
\text { technology } \\
\text { center }\end{array}$ \\
\hline $\begin{array}{l}\text { test } \\
\text { time(hour) }\end{array}$ & 2500 & 1000 & 2000 \\
\hline $\begin{array}{l}\text { leaked } \\
\text { condition }\end{array}$ & no leakage & no leakage & no leakage \\
\hline
\end{tabular}

Table 1: Seal test results

After the statistics of the end of the thermostat cover leakage failure for the average mileage is $41148 \mathrm{~km}^{[9]}$. If the statistics of vehicle travel data, according to the daily work of 8 hours per car, speed $80 \mathrm{Km} / \mathrm{h}$, a month on the driving $19200 \mathrm{Km}$. And then consider the commercial vehicle assembly, testing, a sale is about three months or so the end of the thermostat cover leakage phenomenon can be reflected from the customer. After tracking for three months, the service site did not find the pump leak return. And the statistics of the improved one year after the return of the three packages, the model pump did not appear the same temperature of the water heater cover the end of the water leakage phenomenon. It can be concluded that the model of the pump thermostat cover 
end leakage failure mode elimination, which improved after the effect is good. At the same time this improvement reduces the type of pump after-sales compensation amount, improve the competitiveness of the product.

\section{Conclusion}

Through the analysis of the dismantling of the pump failure pieces, the cause of the leak is statistically analyzed. The leakage of thermostat cover accounts for a relatively high proportion. This paper analyzes the causes of the leakage fault from the processing dimension, sealing material and design structure. This offers some improvement measures for solving the leakiness of the pump assembly. Eventually we found the main problem is the aging of rubber. For the aging of the rubber to improve the design, this problem is analyzed and discussed from three aspects. By taking into account cost issues, operational problems and other issues, it is found that by adding a gasket is a viable option. Paper pad or foam rubber metal composite plate gasket can be increased. By taking into account operational problems, it is possible to increase the foamed rubber metal composite sheet gasket. The size of the foamed rubber metal composite panel gasket was designed while the sealed face pressure was checked. The final seal test and market product inspection showed that the pump thermostat cover end of the leak did not leak, the improvement effect was obvious.

\section{References}

[1] Cao Zhanlong, Li Hongyan, Ren Bolin . "Failure analysis and improvement for automotive water pump", Journal of Hubei University of Automotive Technology, 2013, 27( 3) : 65 - 68.

[2] Li Xinglin, Wang Chengtao, Cao Maolai, et al. "Tests and analysis of automotive water pump bearing seal performance. Bearing”, 2000 (6): 33 35.

[3] Liu Tingting, Wang Tong, Yang Bo , et al. "Numerical simulation and structure improvement for a car pump with opened centrifugal impeller", Journal of engineering thermo physics, 2009, 30 (6): $61-63$.

[4] Zhang Junjie, Shi Weidong, Zhang Desheng, et al . " Numerical simulation and optimization of automotive water pump to eliminate shaft seal failure in high speed operation", Journal of drainage and irrigation machinery engineering, 2015, 33( 7) : 606-610.

[5] Zhou Qiong, Li Zhengmei, Tang Jianping, et al . "Finite element analysis of contact stress of automotive water pump bearing's seal", Bearing, 2011 (1): $4-7$.

[6] Kong Weifeng, Li Lingjun, Li Pengyong, et al . "Dynamic characteristics of automobile pump assembly",Journal of mechanical strength, 2011, 33 ( 5 ) : $767-770$.
[7] Yuan Shouqi, Zhang Tingting, Zhang Jinfeng, et al. "Optimization design and test study on automobile cooling pumps", Journal of drainage and irrigation machinery engineering, 2014, 32( 2): $93-97$.

[8] Zhang Gang. "The research and development of the automobile engine cooling system test bench", Wuhan University of Technology, 2012.

[9] Duan Liping, Yuan Tao, Chen Yun, et al. "Failure analysis of water pump assembly of a certain diesel engine", Ordnance material science and engineering, 2008, 31( 1) : $71-74$. 